L.

\title{
Ueber die Zusammensetzung der Asche von Kartoffelknollen.
}

\author{
Von \\ Dr. J. Moser, \\ Professor der Chemie und Physik an der k. k. höhern landwirth- \\ schaftlichen Lehranstalt zu Dngrisch-Altenburg.
}

(Im Auznge a. d. Sitzungsber. d. kais. Akad. d. Wiss. Bd. XI. III. Heft. Octbr.)

Die untersuchten zwei Sorten von gesunden Kartoffelknollen stammten aus der Lombardie.

Die Einäscherung der sorgfältig gereinigten, zerschnittenen und in Steinguttiegeln verkohlten Knollen geschah nach Dr. Strecker's Angabe in der Muffel bei möglichst niedriger Temperatur, wodurch eine wohl nicht völlig kohlenfreie Asche erzielt wurde, dagegen aber auch kein Verlust an Chloriden u. s. w. eintreten konnte. Die letzten Antheile der Kohle in der Asche liessen sich weder durch fortgesetzte Einwirkung einer sehr gelinden Rothglut in der Muffel, noch durch Anwendung stärkerer Glühhitze über der Spirituslampe entfernen; und weil in letzterem Fall immer ein auf Verluste an Chloriden und Schwefelsäure deutendes Zusammenbacken der Asche eintrat, so wurde die rückständige Kohle bei der Asche gelassen, und ihre Menge durch Zurückwägen bestimmt.

Die Asche wurde mit.verdünnter Salpetersäure bei $50-60^{\circ} \mathrm{C}$. über eine Stunde digerirt, und die rückständige Kohle auf einem kleinen Filtrum gesammelt und mit heissem, Wasser ausgewaschen. Im Filtrate wurden Chlor, Schwefelsäure und Kieselsäure auf die gewöhnliche Art abgeschieden. Zur Bestimmung der Phosphorsäure, welche in der Ueberschuss an Alkalien enthaltenden Asche nur als dreibasige Säure vorkommt, wurde der in den Laboratorien von Liebig, Fresenius u. A., derzeit bei der Analyse von Pflanzenaschen verfolgte Gang gewählt, welcher sich auf die Unlöslichkeit des phosphorsauren Eisenoxydes

Journ. t. prakt Chomio. LXI. 6. 
$\left[2 \mathrm{Fe}_{2} \mathrm{O}_{3} 3 \mathrm{PO}_{5}\right]$, so wie des oxalsauren Kalkes in Essigsäure, und auf die Löslichkeit des phosphorsauren Calcium- und Magniumoxydes in derselben Säure stützt. Es wurde desshalb das von der abgeschiedenen Kieselsäure erhaltene Filtrat erhitzt, mit Ammon fast neutralisirt und dann mit essigsaurem Ammon im Ueberschusse versetzt, um das phosphorsaure Eisenoxyd abzuscheiden. Ist die Flüssigkeit hinreichend verdünut und heiss, so hat man nicht zu besorgen, dass mit dem phosphorsauren Eisenoxyde auch ein Theil der phosphorsauren alkalischen Erden sich abscheidet, was stets erfolgt, wenn die Flüssigkeit concentrirt ist. Das essigsaure Filtrat vom phosphorsauren Eisenoxyd wurde zur Abscheidung des Kalkes mit Oxalsïure oder oxalsaurem Ammon versetzt und der Niederschlag nach 24 Stunden filtrirt. Das Filtrat, welches noch freie Essigsäure enthalten muss, gab mit Ammon übersättigt die Magnesia sammt einem Theil der Phosphorsäure; der noch im Filtrat enthaltene Rest der Phosphorsäure wurde durch schwefelsaure Magnesialösung (die mit Chlorammonium versetzt ist), ausgefällt.

Zur Trennung der überschüssig zugesetzten schwefelsauren Magnesia von den Alkalien diente essigsaurer Baryt; die Alkalien wurden als Chloride durch Platinchlorid getrennt, und ward auch das Chlornatrium durch direkte Wägung bestimmt, so dass nur die Kohlensäure aus dem Verluste zu berechnen kam; übrigens wurden auch direkte Kohlensäurebestimmungen gemacht, deren Ergebnisse mit den berechneten gut stimmend - unter den Resultaten folgen.

Bezüglich der rïckständigen Kohle ist noch zu erwähnen, dass dieselbe, nachdem ihr Gewicht bestimmt war, sammt dem Filter eingeäschert wurde. Die geringe Menge dieser meistens lichtsrauen und sandfreien Asche wurde derjenigen Flüssigkeit zugesetzt, die behufs der Kieselsäureabscheidung eingedampft wurde. 


\section{Resultate der Analyse:}

1. Gehalt an Wasser und an Trockensubstanz.

Die Knollen der Sorte Nr. I enthielten in 100 Theilen:

76,027 p. C. Wasser und

23,973 " "Trockensubstanz.

Die Knollen der Sorte $\mathrm{Nr}$. II enthielten in 100 Theilen:

80,135 p. C. Wasser und

19,865 ", Trockensubstanz.

2. Aschengehalt der Knollen.

Derselbe beträgt (nach Abzug der Kohle)

bei Nr. I 1,039 p. C. der frischen oder 4,334 p. C. der " II 1,151 " " $\quad, \quad \operatorname{oder} 5,798$ p. C. der trockenen Substanz

Wird von diesen Zahlen noch der Gehalt der Aschen an Kohlensäure abgerechnet, um aus der nachfolgenden Zusammenstellung (3) die Erschöpfung des Bodens an Mineralsubstanz direkt berechnen zu können, so ergiebt sich ein

Aschengehalt nach Abrug der Kohlensäure:

bei Nr. I von 0,919 p. C. der frischen oder 3,846 p. C. der " "II " $1,045, \quad$ " " $\quad \begin{array}{r}\text { trockenen Substanz } \\ \text { oder 5,178 p. C. der } \\ \text { trockenen Substanz. }\end{array}$

3. Procentische Zusammensetzung der Asche (mit Ausschluss der Kohlensäure).

In 100 Theilen Asche sind enthalten:

\begin{tabular}{|c|c|c|}
\hline & & \\
\hline lorkali & 1,098 & 4,110 \\
\hline Chlornatrium & 2,914 & 3,085 \\
\hline Kaliumoxyd & 60.561 & 63,550 \\
\hline Eisenoxyd & 0,79 & 0,453 \\
\hline Kalk & $1=09$ & 1,803 \\
\hline Magnesia & 3,60 & 4,261 \\
\hline Phosphorsäur & 817,5 & 18,578 \\
\hline hwefelsžure & 4,55 & 3,920 \\
\hline $\begin{array}{l}\text { Kieselsăur } \\
\text { Mangan }\end{array}$ & & $S p$ \\
\hline & & \\
\hline
\end{tabular}

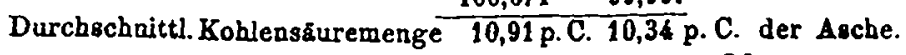


Alle diese Zahlen sind ein Mittel von mindestens je zwei gut stimmenden Daten. Bezüglich der sub 1 und 2 angeführten ist zu erwähnen, dass die Knollen ungeachtet des weiten Transportes ganz frisch aussahen, dass also die Bestimmungen über den Wassergehalt wenigstens nahezu richtig sind.

Dem äussern Ansehen nach unterschieden sich diese Knollen nur dadurch, dass Nr. I eine etwas hellere Schale hatte; bei Nr. If zeigten sich im Innern blassrothe Ringe.

Beide Sorten gehören zu den feinschaligen Kartoffeln. Während des Winters wurden die Knollen in einem ebenerdigen etwas feuchten Locale aufbewahrt, erhielten sich daselbst ganz frisch, und hatten sämmtliche Knollen gegen Ende März reichlich Keime getrieben.

Zur Vergleichung der Resultate sind die eben angeführten Analysen mit denen von Boussingault, Way und Herapath in der nachfolgenden Tabelle I zusammengestellt. Bei der Abhängigkeit der Resultate von den Methoden der Untersuchung erschien es wichtig, die Daten der zwei letztgenanuten Analytiker aufzuführen, weil sie mehrere Untersuchungen anstellten, die wenigstens unter sich vergleichbar sind oder sein sollen. Boussingault's Arbeit wurde mit aufgenommen, weil sie am häufigsten citirt und zu weitern Berechnungen benutzt wird. 
Ton Kartofelknollen.

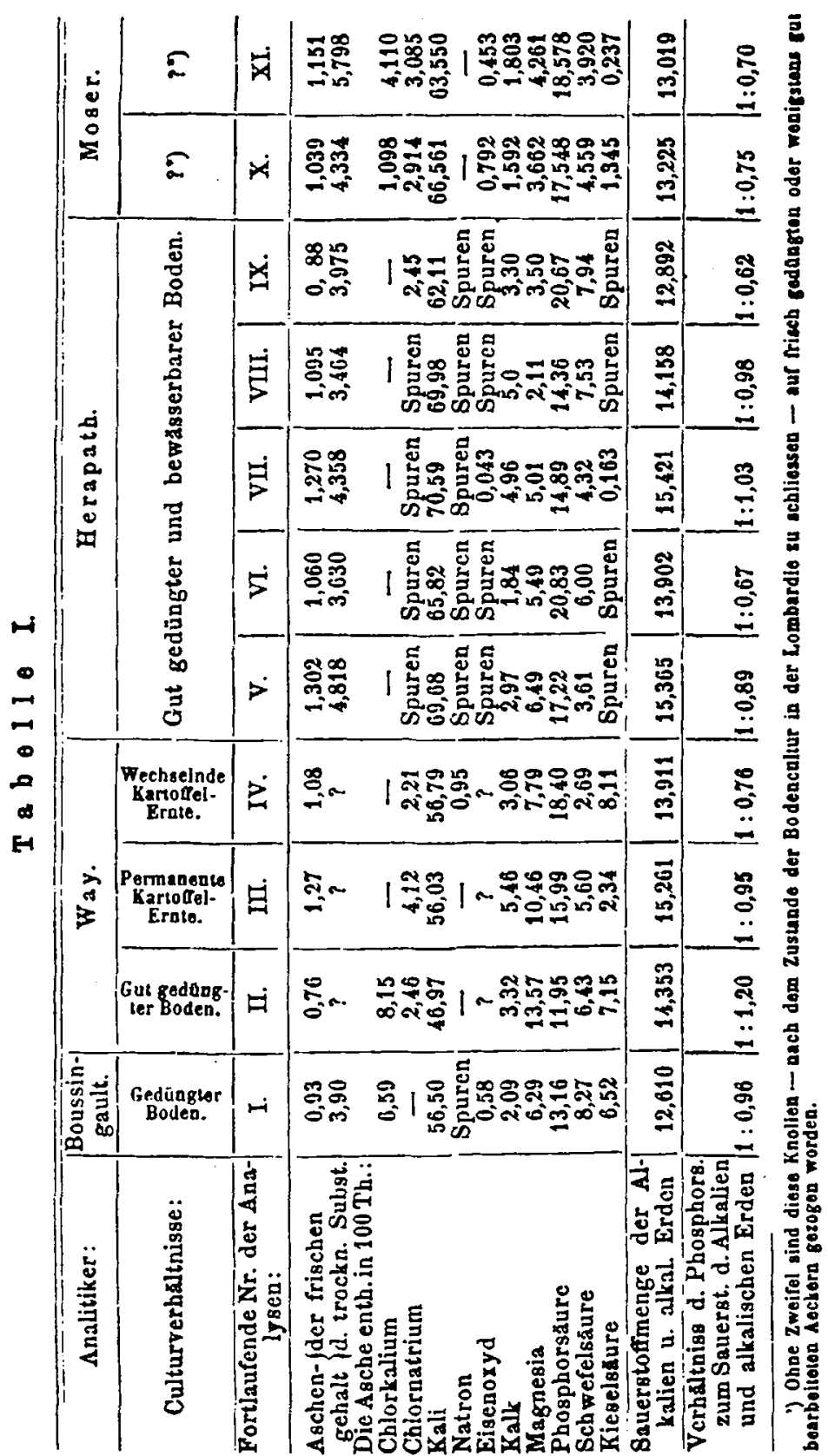


Die in dieser Tabelle neben einander stehenden Zahlen zeigen auffallende Vorschiedenheiten sowohl in der Aschenmenge als auch in der Qualität und Quantitït der Aschenbestandtheile. Wie die vorletzte Querspalte ausweist, ist der Versuch, diese Verschiedenheiten wenigstens bezüglich der Basen in eine Uebereinstimmung zu bringen, nicht sehr günstig ausgefallen; ein Gleiches ist der Fall in Bezug auf das in der letzten Querspalte angeführte Verhältniss der Phosphorsäure zum Sauerstoff der Alkalien und alkalischen Erden.

Die Verschiedenheiten obiger Angaben in den qualitativen und quantitativen Verhältnissen von Chlorkalium, Chlornatrium und Kiıselsäure finden wohl darin eine Erklärung, dass die in Boden sich entwickelnden Knollen von den leicht löslichun Chloriden der Alkalien, je nachdem diese in grösserer odir geringerer Menge vorhanden sind, auch wechselnde Mengen aufnehmen, während die Kieselsäure ebensowohl auf diese Art, sowie überdem noch als zufallige Verunreinigung in die Asche gelangen bann; es wird daher der Schluss nicht gewagt sein, dass diese Bestandtheile in der Asche der Kartoffelknollen nicht $\mathrm{zu}$ den wesentlichen gehören, ein Aehnliches dürfte vom Eisenoxyd, dessen Menge von ausien gegen innen (wie ich mich mehrfach überzeagte) beträchtlich abnimmt. Lässt man nun diese als nicht wesentlich $\mathrm{zu}$ betrachtenden Bestandtheile der Asche aus den in der Tabelle I. angeführten Analysen weg, und berechnet dann aus dem Reste wieder die procentische Zusammensetzung der Asche, so ergeben sich die in der Tabelle II. zusammengestellten Zahlen: 
von Kartoffelknollen.

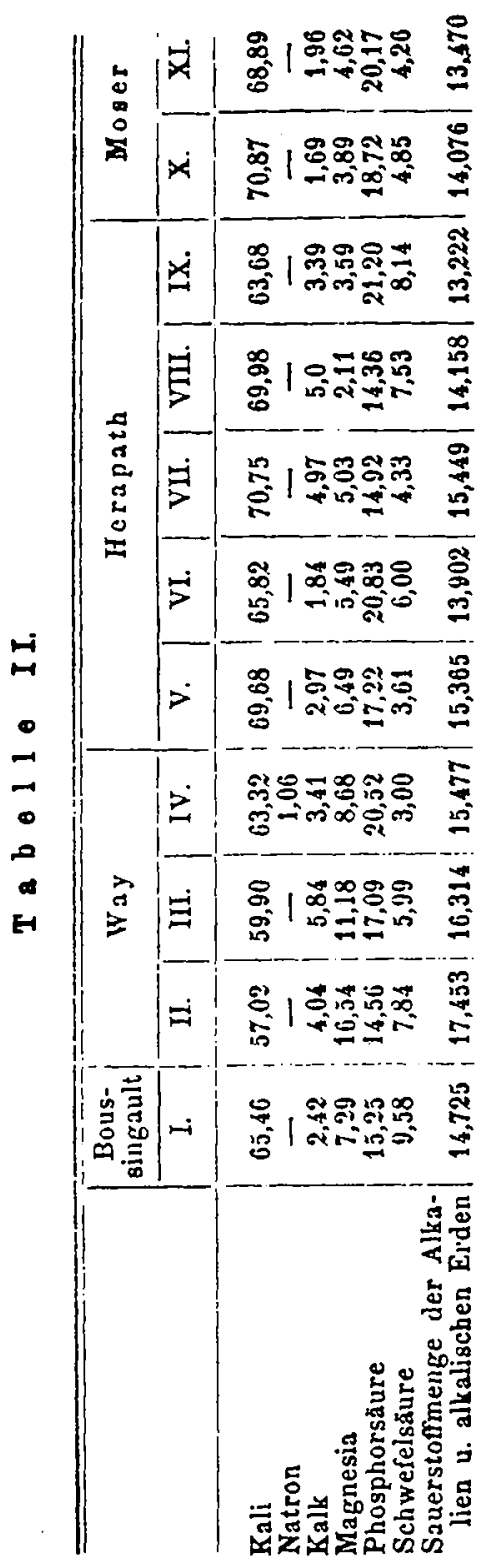


Durch diese Umrechnung werden die quantitativen Angaben der einzelnen Analytiker über das Kaliumoxyd und die Phosphorsäure bedeutend näher gerückt, während das relative Verhältniss des Kali zu Kalk und Magnesia, so wie das der letztiren zwei unter sich dasselbe bleibt. Die Unterschiede in der Sauerstoffmenge der Basen werden noch zufällig grösser, weil sie proportionirt mit den eliminirten Zahlen steigen; das Verhältniss der Phosphorsäure zum Sauerstoff der Basen bleibt dagegen wieder dasselbe, wie in der Tabelle I. Wenn auch diese letzte Tabelle noch beträchtliche Differenzen ausweist, so dürften die darin zusammengestellten Zahlen doch Anhaltspunkte zu quantitativen Mischungsverhältnissen geben, behufs der Ausführung von synthetischen Versuchen, die uns über die, die Aschen der Pa:anzen betreffenden Fragen wohl nur allein genügenden und sichern Aufschluss geben können.

\section{LI. \\ Untersuchung von Ackererden aus dem Banate. \\ Von \\ Radolph Ritter von Hanor.}

(Im Auszuge aus dem Jahrb. d. k. k. geologischen Rcichsanstalt. 3. Jahrg. 18.j2. IV. Vierteljahr. S. 81.)

Die durch ihre ausserordentliche Ertragsfähigkeit für landwirthschaftliche Culturpflanzen aller Art, insbesondere für Cerealien und Oelfrüchte, so sehr berühmten Bodenarten des Banates haben schon lange den Wunsch nach einer wissentschaftlichen Untersuchung derselben rege gemacht. Der Verf, hat eine solche im Laboratorio der k. k. geologischen Reichsanstalt ausgefuhrt.

Die Erden wurden an verschiedenen weit von einander entlegenen Punkten des Banates mit grosser Sorgfalt ge- 